\title{
SARS-CoV-2 Infection in Children and Kawasaki Disease. What Is the Relationship?
}

\author{
Fariba Shirvani ${ }^{1, *}$ and Asieh Mosallanejad ${ }^{2}$ \\ ${ }^{1}$ Pediatric Infections Research Center, Research Institute for Children Health, Shahid Beheshti University of Medical Sciences, Tehran, Iran \\ ${ }^{2}$ Imam Hossein Hospital, Shahid Beheshti University of Medical Sciences, Tehran, Iran \\ "Corresponding author: Pediatric Infections Research Center, Research Institute for Children Health, Shahid Beheshti University of Medical Sciences, Tehran, Iran. Email: \\ shirvanifariba@rocketmail.com \\ Received 2020 June 27; Accepted 2020 June 28.
}

Keywords: Child, Kawasaki Disease, COVID-19

\section{Dear Editor,}

Severe acute respiratory syndrome coronavirus 2 (SARS-CoV-2) is a novel virus affecting adults and children. The spread of severe acute respiratory syndrome resulted in a global pandemic by SARS-CoV-2. The pediatric population is responsible for a smaller proportion, and $2 \%$ of cases are described under age 20 (1). Whether this ratio is a sign of low sensitivity to the disease in children vs. adults or a higher rate of asymptomatic cases is unclear. Recently, attention has been drawn to the link between Kawasakilike disease and coronavirus disease-2019 (Covid-19) in children (2). Kawasaki disease (KD) initially described in 1967 in Japan is a multisystem inflammatory syndrome, typically occurs in six months to five years old children. Tomisaku Kawasaki stated that there may be a correlation between viral infection and a cluster of patients with "Mucocutaneous Lymph Node Syndrome", 40 years later, Espar et al. found eight coronavirus-positive nasal swabs among eleven patients with KD (3). Chang et al. have shown that viruses, including coronavirus, has been linked to $\mathrm{KD}$ (4). Recently, there is an increasing occurrence of KD potentially linked with coronavirus between children in a high-risk area (5). Cases of multisystem inflammatory syndromes and Kawasaki occurred in Europe and the USA named pediatric inflammatory, multisystem syndrome temporally associated with SARS-CoV-2 infection (PIMS-TS), and multisystem inflammatory syndrome in children (MIS-C), respectively (6).

A cluster of 8 children with features of atypical KD, toxic shock syndrome (TSS), and KD shock syndrome (KDSS) were reported in South Thames retrieval services pediatric ICU during 10 days in mid-April 2020. Laboratory feature of these children was hyperferritinemia, ele- vated proBNP, Procalcitonin, and CRP. Most of them were Afrocaribian and were treated with inotropes, hydrocortisone, and IVIG. SARS-CoV-2 was positive in two cases, one of whom died (7). After the outbreak started in February 2020, Italy, the first affected European country, experienced a high incidence of Kawasaki-like disease in children. In Bergamo city with the highest rate of death and infection in Italy, 10 cases of newly diagnosed KD (first group) were compared with 19 cases of $\mathrm{KD}$ in the last five years (second group). The incidence of KD was 30 times higher in cases admitted from February 18 to April 20, 2020, in contrast to cases admitted from January 1, 2015, to February 17, 2020 in which 8/10 of first group cases were positive for IgG and 3/10 were positive for IgM too. Mean age was significantly higher in the first group; cardiac involvement occurred in 2/19 in second and 6/10 in the first group. Kawasaki disease shock syndrome (KD with hemodynamic instability) and macrophage activating syndrome (MAS) occurred in 0/19 in the second and 5/10 in both KDSS and MAS cases in the first group. The first group had significantly lower WBC, lymphocyte, and platelet count. The first group included more severe cases with atypical KD and they required more adjunctive treatment (8). In response to this cluster in the UK, the emergence of an unusual disorder was notified to National Health Services on April 25. Owing to the rise in antibodies and little evidence of viral replication in most patients, the mechanism of MIS-C seems to be antibody or immune complex-mediated (2). Given the recent increase in MIS-C or PIMS-TS in the final stage of the first epidemic of SARS-CoV-2, it is important to warn about the possibility of its occurrence in children. However, its prevalence is low in children equivalent to one in 1,000 children exposed to SARS-CoV-2 (8), early detection 
and appropriate treatment with a corticosteroid, IVIG, Inotropes, and other modalities are important.

\section{Footnotes}

Authors' Contribution: Fariba Shirvani designed the letter and wrote the draft and Asieh Mosallanejad edited it.

Conflict of Interests: There was no conflict of interest.

Funding/Support: There was no financial support.

\section{References}

1. Wu Z, McGoogan JM. Characteristics of and Important Lessons From the Coronavirus Disease 2019 (COVID-19) Outbreak in China: Summary of a Report of 72314 Cases From the Chinese Center for Disease Control and Prevention. JAMA. 2020. doi: 10.1001/jama.2020.2648. [PubMed: 32091533].

2. Viner RM, Whittaker E. Kawasaki-like disease: emerging complication during the COVID-19 pandemic. Lancet. 2020;395(10239):1741-3. doi: 10.1016/S0140-6736(20)31129-6. [PubMed: 32410759]. [PubMed Central: PMC7220168].
3. Calabri GB, Formigari R. Covid-19 and Kawasaki Disease: A Glimpse at the Past for a Predictable Future. Pediatr Cardiol. 2020;41(5):1075. doi: 10.1007/s00246-020-02385-0. [PubMed: 32462467]. [PubMed Central: PMC7250736].

4. Chang LY, Lu CY, Shao PL, Lee PI, Lin MT, Fan TY, et al. Viral infections associated with Kawasaki disease.JFormos Med Assoc. 2014;113(3):148-54 doi:10.1016/j.jfma.2013.12.008. [PubMed: 24495555]. [PubMed Central: PMC7125523].

5. Jones VG, Mills M, Suarez D, Hogan CA, Yeh D, Segal JB, et al. COVID19 and Kawasaki Disease: Novel Virus and Novel Case. Hosp Pediatr. 2020;10(6):537-40.doi:10.1542/hpeds.2020-0123. [PubMed:32265235]

6. Toubiana J, Poirault C, Corsia A, Bajolle F, Fourgeaud J, Angoulvant F, et al. Kawasaki-like multisystem inflammatory syndrome in children during the covid-19 pandemic in Paris, France: prospective observational study. BMJ.2020;369:m2094. doi:10.1136/bmj.m2094. [PubMed: 32493739].

7. Riphagen S, Gomez X, Gonzalez-Martinez C, Wilkinson N, Theocharis P. Hyperinflammatory shock in children during COVID-19 pandemic. Lancet. 2020;395(10237):1607-8. doi: 10.1016/S0140-6736(20)31094-1. [PubMed: 32386565]. [PubMed Central: PMC7204765].

8. Verdoni L, Mazza A, Gervasoni A, Martelli L, Ruggeri M, Ciuffreda M, et al. An outbreak of severe Kawasaki-like disease at the Italian epicentre of the SARS-CoV-2 epidemic: an observational cohort study. The Lancet. 2020;395(10239):1771-8. doi:10.1016/s0140-6736(20)31103-x. 